\title{
Stockage de l'eau pour l'irrigation
}

\author{
A. Roux, P. Faure \\ Ministère de l'Agriculture et de la Forêt, \\ Direction de l'Espace rural et de la Forêt
}

\section{Introduction}

Les deux épisodes de sécheresse consécutifs que vient de connaître la France ont montré la sensibilité de l'activité agricole à l'insuffisance de l'apport d'eau. L'eau est indispensable à la croissance des plantes et celle-ci ne peut être trouvée que dans le sol qu'elle vienne de la pluie, de nappes sous-jacentes, ou d'un apport d'irrigation.

Dans beaucoup de régions françaises, pendant la période estivale, les réserves en eau des sols s'amenuisent au point que l'apport d'eau d'irrigation est une condition sine qua non pour qu'existe une agriculture rentable, ou du moins, plus facilement adaptable aux exigences des marchés agricoles et du développement économique des terroirs.

Même si ces aspects favorables doivent, dans un certain nombre de cas, être nuancés, il n'est pas étonnant que l'on constate une extension des surfaces irriguées, dont la moitié au moins provient d'initiatives privées inorganisées. L'augmentation des capacités de stockage de l'eau pour l'irrigation est de ce fait nécessaire en raison d'une part du décalage temporel qui existe entre les périodes de forte pluviométrie (automne, hiver) et celles où le prélèvement d'eau est le plus intense (juillet, août), et d'autre part, du caractère de consommation nette, sans retour au milieu naturel, que constituent les arrosages pendant les périodes de faibles écoulements.

Le présent exposé a pour but de faire le point sur l'adéquation actuelle entre les ressources et les besoins et de montrer qu'il existe des limites écologiques et économiques au développement de l'irrigation qui auront une influence certaine sur le volume de stockage à créer ou à mobiliser à partir de réserves existantes.

\section{Etat des lieux}

D'après les données fournies par les Agences de Bassin, reprises chaque année dans le bilan de "l'Etat de l'Environnement ", les prélèvements d'irrigation représentent 4 milliards de $\mathrm{m}^{3}$ par an, à comparer aux 17 milliards de $\mathrm{m}^{3}$ utilisés par les Centrales Electriques, 6 milliards de $\mathrm{m}^{3}$ pour l'industrie et 5 milliards de $\mathrm{m}^{3}$ pour la distribution d'eau potable.

A priori, ils apparaissent faibles et ne pas poser de problème particulier si on les compare aux 175 milliards de $\mathrm{m}^{3}$ qui constituent les ressources écoulées annuellement sur le territoire français.

Mais si l'on raisonne en terme de consommation nette, c'est-à-dire de quantité soustraite au milieu naturel sans restitution plus ou moins rapprochée, dans le temps ou dans l'espace, l'irrigation représente aujourd'hui $44 \%$ du total, loin devant les autres utilisateurs.

On comprend donc les attaques souvent vives que les milieux écologiques adressent à l'égard de l'irrigation, accusée de dégrader les milieux naturels.

Pour apprécier les besoins d'irrigation et leur évolution, il faut se placer à deux échelles différentes :

- tout d'abord au niveau des besoins à l'hectare: les apports varient dans des proportions importantes suivant les cultures, les années et les régions à l'intérieur d'une fourchette allant de 1000 à $3500 \mathrm{~m}^{3}$ pour les réseaux modernes sous pression. Toutefois si on intègre les pertes constatées au cours du transport et de la distribution on doit élargir la fourchette jusqu'à $7000 \mathrm{~m}^{3}$ voire $20000 \mathrm{~m}^{3}$ dans certains réseaux gravitaires du sud-est dont la réhabilitation est heureusement actuellement en cours ;

- ensuite au niveau des superficies irriguées : la surface irriguée a plus que doublé dans les vingt dernières années, passant de 500000 ha en 1970 à 1200000 ha en 1989 . L'augmentation annuelle depuis une dizaine d'années se situe autour de 40000 ha.

Mais cette évolution globale masque des disparités régionales fortes; en effet, si la surface irriguée des régions méditerranéennes (Midi méditerranéen, Sud-Est et Corse) est restée stationnaire, d'autres régions ont connu des croissances fortes, comme le montre le tableau ci-dessous :

Evolution des superficies irriguées

\begin{tabular}{|l|c|c|c|}
\hline \multirow{2}{*}{ Région } & \multicolumn{3}{|c|}{ Surfaces irriguées (1 000 ha) } \\
\cline { 2 - 4 } & 1970 & 1988 & $\begin{array}{c}\text { variation } \\
\%\end{array}$ \\
\hline Centre & 66,8 & 149,5 & 124 \\
Pays de Loire & 22,9 & 76,2 & 232 \\
Poitou-Charentes & 8,0 & 98,5 & 1130 \\
Aquitaine & 67,7 & 229,7 & 240 \\
Midi-Pyrénées & 60,5 & 210 & 247 \\
Alsace & 6,8 & 33,6 & 394 \\
Rhône-Alpes & 36,8 & 76,5 & 108 \\
\hline
\end{tabular}


Enfin il convient de remarquer que la superficie irriguée française ne représente qu'environ $4 \%$ de la SAU, pourcentage qu'il faut comparer à celui de nos voisins et concurrents méditerranéens : Espagne, Italie et Grèce.

\begin{tabular}{|l|c|c|}
\hline & $\begin{array}{c}\text { Superficies totales } \\
\text { irriguées en ha }\end{array}$ & $\begin{array}{c}\% \\
\text { SI/SAU }\end{array}$ \\
\hline Espagne & 3300000 & 12 \\
Italie & 3600000 & 21 \\
Grèce & 1200000 & 21 \\
\hline
\end{tabular}

De l'examen de ces données concernant l'irrigation, on en déduit que la question du stockage des eaux agricoles se pose de manière différente selon les régions. On peut en première approximation distinguer trois types de situation :

1. Dans les régions Provence-Alpes-Côte d'Azur, Languedoc-Roussillon et Corse l'irrigation est ancienne, voire très ancienne; les ressources existent soit sous forme de barrages importants hydroélectriques où des réserves agricoles ont été négociées en leur temps (Serre-Ponçon, les barrages du Verdon) ou de prises d'eau sur des rivières à fort débit (Rhône, Durance). Dans ce contexte, l'extension des surfaces irriguées passe par une augmentation marginale des volumes stockés mais surtout par une modernisation des réseaux de transport et de distribution et une réaffectation majoritaire des volumes ainsi sauvegardés à l'agriculture.

2. Dans les régions Aquitaine et Midi-Pyrénées, où le développement de l'irrigation est moins chargé d'histoire que dans les régions précédemment citées, la création de réserves n'a pas suivi le rythme d'accroissement des besoins et une ambitieuse politique de soutien des étiages des rivières est actuellement mise en place. Sa description fera l'objet d'un exposé particulier par J.-L. ReDAud.

3. Dans les autres régions de forte croissance des superficies irriguées, l'analyse doit être menée au cas par cas et conduira dans la plupart des contextes, à initier des stratégies ambitieuses de gestion des milieux aquatiques alliant l'augmentation à l'amélioration de la ressource. Ainsi, par exemple :

- en Poitou-Charentes, le développement de l'irrigation très tardif s'est fait de manière individuelle souvent à partir de forages ou de prises en rivière rendant indispensable une amélioration de la gestion collective des besoins et la création de ressources nouvelles pour éviter la concurrence avec l'usage eau potable pour les eaux souterraines et l'abaissement des débits d'étiage au-delà de limites inacceptables ;

- en Alsace les irrigants disposent d'une nappe souterraine puissante et les difficultés ne résultent pas de la quantité disponible mais des conséquences sur la qualité ;

- en Rhône-Alpes l'accroissement des surfaces irriguées pose surtout problème dans le Sud où la création de ressources supplémentaires est indispensable.

\section{Facteurs d'évolution}

Après cet état des lieux technique, il convient de s'interroger sur les déterminants qui influencent le développement de l'irrigation aujourd'hui.

On peut les ranger en trois catégories selon que leur impact est positif, négatif ou encore inconnu à l'heure actuelle.

Parmi les facteurs positifs qui poussent les agriculteurs à recourir à l'irrigation, on peut citer :

- l'accroissement de la compétitivité des exploitations agricoles ;

- la régularisation de la production ;

- la possibilité de diversifier les cultures;

- l'amélioration de la qualité des produits.

Même si, comme on le verra plus loin, ces aspects favorables doivent être parfois nuancés, ils constituent pour l'exploitant agricole un ensemble de raisons largement suffisantes pour l'inciter à s'équiper et rendre les revendications pour l'accès à l'irrigation pressantes dans beaucoup d'endroits.

Les collectivités locales jouent également un rôle important dans le développement de l'irrigation. Pour certaines régions, le maintien de l'occupation de l'espace rural et d'un tissu économique vivant passe par l'aide à l'agriculture. Il s'agit donc pour les élus locaux d'une action d'aménagement du territoire qui évite le retour à la friche et toutes les conséquences néfastes qui en découlent.

Par contre il existe également des facteurs défavorables au développement de l'irrigation et donc à la construction de nouveaux stockages d'eau :

- les contraintes de gestion des milieux aquatiques vont aller en augmentant. En effet, au regard notamment des impacts négatifs de certains aménagements hydroagricoles, les revendications du public pour une meilleure prise en compte de l'environnement ont su se faire jour et imposer une place plus importante que dans le passé à la préservation du milieu naturel en obligeant à des études justificatives plus poussées. La situation rencontrée dans le sud-ouest français par le PDRE préfigure ce que sera demain le cas général. Cet exemple vous sera exposé plus longuement par J.-L. REDAUD ;

- au niveau micro-économique le recours à l'irrigation ne s'avère pas toujours bénéfique. Il nécessite des investissements importants et les intrants deviennent plus coûteux. Il en résulte un accroissement des charges de l'entreprise et celle-ci supporte donc des risques plus importants, en particulier en cas de défaillance du réseau d'irrigation comme en 1989 et 1990 ;

- au niveau macro-économique, même si l'affirmation que l'irrigation ne fait qu'aggraver la création d'excédents agricoles doit être nuancée, il n'en reste pas moins que le développement des surfaces irriguées dans une zone donnée peut contribuer à délocaliser certaines productions et l'enrichissement des uns peut entraîner un relatif appauvrissement des autres.

Plus difficile à évaluer sur la vitesse de développement de l'irrigation sont l'impact des deux négociations actuel- 
lement en cours portant sur le GATT et la nouvelle Politique agricole commune.

Les éléments connus à l'heure actuelle sont encore insuffisants pour en déduire les réactions vis-à-vis de l'irrigation que vont susciter auprès des agriculteurs les nouvelles modalités de fixation des prix et d'aides au soutien des revenus.

Toutefois quand on considère la faiblesse de la superficie irriguée en France et le fait qu'une grande partie des cultures irriguées (la presque totalité dans le sud-est) porte sur des productions non soutenues par la PAC, on peut se demander si l'impact ne sera pas limité. Il faudrait que la nouvelle PAC favorise, par les signaux financiers qu'elle donne à l'agriculteur, les systèmes extensifs pour qu'on assiste à une diminution sensible des surfaces irriguées : ce n'est pas évident dans le cadre des propositions actuelles.

\section{Stratégie actuelle et évolution}

Les graphes joints en annexe montrent les sommes consacrées annuellement depuis 1981 à la constitution de nouvelles ressources en eau (grands barrages et transferts d'intérêt national, moyens, et petits barrages et retenues collinaires relevant des financements régionalisés).

Il importe de remarquer que la dépense globale concernant l'ensemble des ouvrages ayant reçu un financement public direct s'est élevée à 3,3 milliards de francs courants TTC sur la décennie 1980 à 89 . Cet effort d'équipement a appelé globalement 1,2 milliard de francs d'aide de l'Etat pour une capacité stockée à usage agricole d'environ 340 millions de mètres cubes.

Les collectivités locales, soit au titre des contrats de plan ou de leur participation aux programmes intégrés méditerranéens apportent une contribution croissante au financement de la construction des grands ouvrages.

Le coût moyen du mètre cube stocké ressort ainsi à près de $8 \mathrm{~F}$ alors que des statistiques plus poussées, et, conduites par catégories d'ouvrages, permettraient d'illustrer une fourchette de coûts allant de $5 \mathrm{~F} / \mathrm{m}^{3}$ pour les grosses retenues, à 10 et $15 \mathrm{~F} / \mathrm{m}^{3}$ pour les petits ouvrages du type collinaire.

Si par ailleurs, comme il vient de l'être évoqué, la capacité de stockage globale réservée à l'irrigation s'est élevée à environ 340 millions de mètres cubes sur $10 \mathrm{ans}$, la consommation agricole a crû de 510 millions de mètres cubes dans le même laps de temps.

Ainsi, malgré l'intensification nette des efforts entrepris depuis 10 à 15 ans, il existe encore un décalage important entre ressources et besoins qui est illustré par les quatre cartes figurant en annexe.

Deux objectifs s'imposent dès lors en vue de la recherche d'une certaine adéquation de l'offre à la demande :

- le premier, et plus urgent, consiste à organiser et à raisonner la demande: recherche d'économies d'eau, de meilleur partage et de meilleure mise à disposition des eaux, incitation à l'engagement contractuel des usagers agricoles à l'aide de règles de gestion et de tarification des eaux adaptées;

- le second, dont les effets sont plus étalés dans le temps, consiste dans l'augmentation des ressources en eau (création ou mobilisation nouvelles), afin de rattraper les retards d'équipements et de permettre de nouvelles expansions contrôlées des besoins.

\section{Petits barrages et retenues collinaires}

Chaque fois que le contexte hydro-géologique le rend possible, la ressource en eau des petits périmètres d'irrigation de quelques centaines d'hectares est mobilisée par des ouvrages de ce type dont le rythme de construction permettait un accroissement annuel des réserves de $9 \mathrm{~m}^{3}$ avant 1986 et permet depuis un accroissement annuel de 35 millions.

Si la multiplication de ces points d'eau apporte une compensation satisfaisante des prélèvements sur le milieu aquatique, elle pose un problème plus global d'évaluation des effets cumulés de ses impacts positifs ou négatifs à l'échelle des bassins et sous-bassins qui commencent à mobiliser décideurs politiques et scientifiques.

\section{Moyens et grands barrages}

Donner la liste de tous les ouvrages en cours de réalisation ou projetés serait longue et fastidieuse. Actuellement les principaux projets concernent le sud-ouest (Plan décennal de développement des ressources en eau dans le bassin Adour-Garonne), les bassins de la Charente, de la Sèvre niortaise et de la Vienne, les Pyrénées-Orientales, le Massif central et la Corse. La carte ci-jointe monte les barrages à usage agicole. Toutefois comme indiqué plus haut cet objectif de rattrapage ne doit pas masquer la nécessité de mieux justifier la nécessité de construction de nouvelles retenues.

Malgré les incertitudes que font peser actuellement les négociations et réflexions en cours sur le nouveau contexte économique dans lequel les chefs d'entreprise agricole devront prendre leurs décisions, il existe une certitude incontournable: on est passé d'une situation où tout hectare supplémentaire équipé pour l'irrigation était un progrès dans le sens du développement économique tant pour l'agriculteur que pour la collectivité à la nécessité d'éclairer les choix tant des points de vue micro et macroéconomique que de l'impact sur l'environnement.

C'est pourquoi, sur toutes les opérations d'une certaine envergure, le Ministère de l'Agriculture et de la Forêt recommande la réalisation d'une étude approfondie de la demande d'irrigation incorporant la comparaison de différents scénarios d'utilisation de l'espace agricole et rural en modes de production plus ou moins intensifiés. Ainsi, sans que la maîtrise des méthodes et des outils soit acquise en totalité à ce jour, ces nouvelles démarches doivent permettre de cerner à la fois, le fonctionnement des processus qui relèvent des lois biophysiques (impacts, risques, résultant de l'action sur les milieux naturels, prélèvements, aména- 
gements, ...) et le fonctionnement de l'exploitation agricole au sein de son environnement local (notamment amont et aval de la production agricole).

L'aide aux choix des opérateurs économiques et financiers ressortira donc de la confrontation entre les techniques et les équipements proposés et les différentes logiques d'action en termes de faisabilité et d'organisation. Il faut donc parler aujourd'hui de développement raisonné de l'irrigation et des ressources en eau. Les équipements qui concourent à rattraper le retard entre besoins et ressources s'imposent, pour les autres il faudra étudier avec soin leur opportunité.

\section{Conclusion}

Pour de nombreuses raisons évoquées dans cet exposé, il n'est pas raisonnable d'envisager un arrêt brutal du déve- loppement des irrigations : la solution aux problèmes qui se posent aujourd'hui au monde agricole ne doit pas être recherchée dans un retour aux pratiques agricoles du bon vieux temps.

Dans beaucoup de nos régions l'agriculture ne peut exister sans utiliser l'eau et la nécessaire compétitivité de nos exploitations exige une disponibilité suffisante de ce facteur de production.

La question fondamentale qui se pose à nous est de savoir si nous pouvons avoir une agriculture performante qui soit plus respectueuse de l'environnement. La même question se pose pour les stockages de l'eau : il nous faut mieux le justifier, progresser dans la réduction de leurs nuisances, développer leurs aspects positifs.

De la réponse qui sera donnée à ces questions dépendra l'intensité du développement du stockage d'eau pour l'usage agricole.

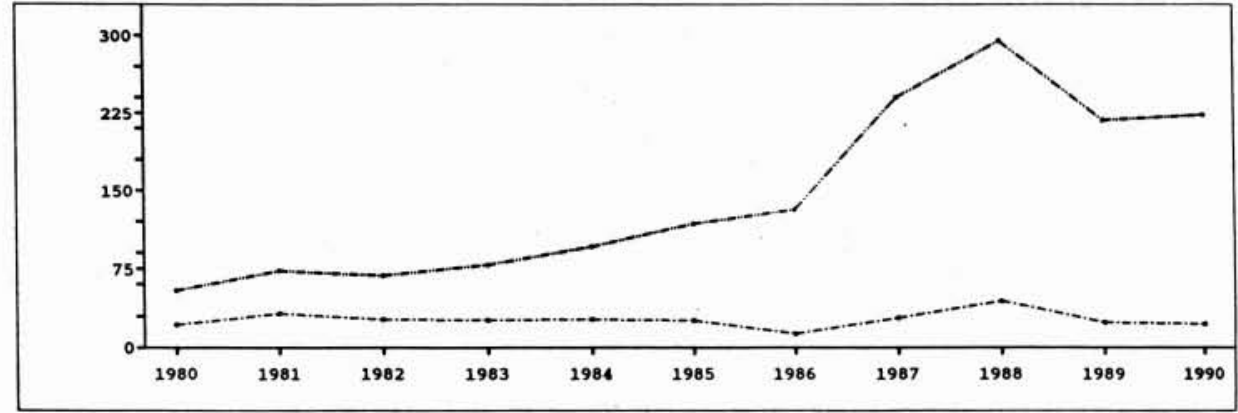

GRANDS BARRAGES (inveatissem. avec aide publique, aide de 1'Etat, en MF 1990)

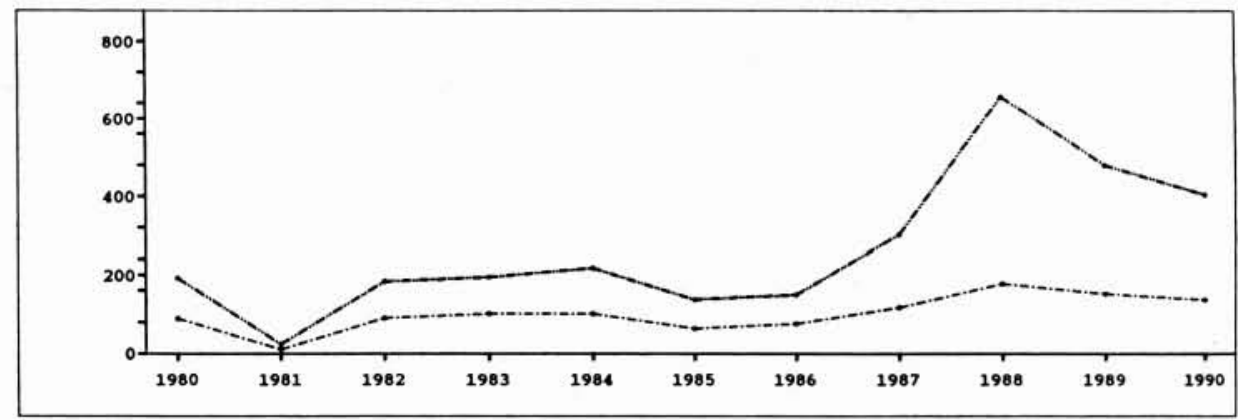

RETENUES COLLITUIRES : VOLUNE STOCKE (en million: de m3)

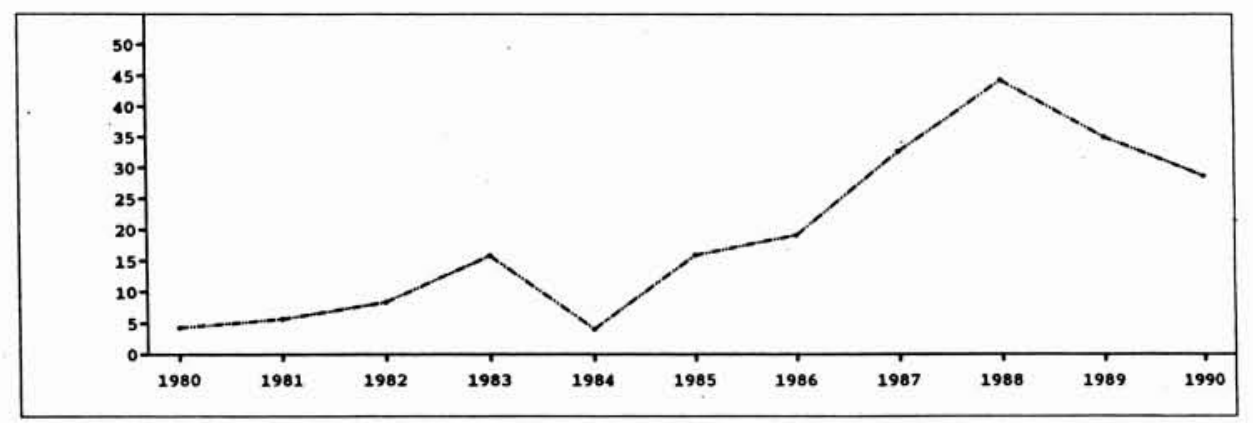

